\title{
La mejora de los logros escolares: la medida y valoración de la inspección educativa
}

\author{
María del Carmen Martínez Serrano ${ }^{1}$ \\ ORCID: 0000-0001-5311-6742
}

\section{Resumen}

El objetivo de la investigación es conocer la valoración que los inspectores de educación tienen sobre su labor en la mejora de los logros escolares del alumnado, a través de la puesta en práctica del plan de inspección, y, de manera más específica, comprobar si existen diferencias en función de determinadas variables personales. La inspección escolar es utilizada en la mayoría de los sistemas educativos europeos como un importante instrumento para el control y promoción de la calidad de las escuelas; no obstante, una revisión pormenorizada de la literatura en relación con la realidad educativa nos evidencia que no son muy frecuentes las investigaciones al respecto. Este artículo pretende aportar información al vacío existente en la investigación educativa en cuanto al estudio de la contribución de la inspección educativa como garante de la calidad y equidad del sistema. Para ello elaboramos un cuestionario (alpha de Cronbach $=, 886$ ) conformado por 27 ítems. El artículo se centra en el primer factor: atención a los miembros de la comunidad educativa. El cuestionario fue cumplimentado por 98 inspectores de las ocho provincias que conforman la Comunidad Autónoma de Andalucía (España). Los resultados constatan que su labor debe centrarse en la atención directa con entrevistas a los miembros de la comunidad educativa. Además, muestran su labor menos efectiva cuando realizan actuaciones relacionadas con el seguimiento de la incidentalidad y los informes homologados.

\section{Palabras clave}

Inspección educativa - Investigación cuantitativa - Educación obligatoria - Mejora educativa.

1- Universidad de Jaén, Jaén, España. Contacto: mcmartin@ujaen.es 


\section{Improving academic success: the measure and assessment from the educational inspection}

\section{Abstract}

This research is an attempt to discern how educational inspectors assess their work on improving the students' academic success, through the implementation of the Inspection Program, and, especially, if there exist differences regarding certain personal variables. To this aim, we have written a questionnaire comprised of 27 items (Cronbach's alpha $=$,886). This paper focuses around the first factor, providing assistance to all members of the educational community. The questionnaire was completed by 98 inspectors from the 8 provinces which make up the Andalusian Autonomous Community (Spain). Results point to the need for the Inspection's work to focus on direct attention to the members of the educational community by means of interviews, and also show how their efforts are less effective when carrying out actions related to isolated incidents or standardized reports.

\section{Keywords}

Educational Inspection - Quantitative research - Compulsory education - Educational improvement.

\section{Introducción}

La educación es determinante en la vida de los individuos, no sólo como espacio de formación social y académica, sino también para su futuro profesional. Atendiendo a los datos recogidos en el Informe Eurostat (EUROSTAT, 2017), en el sistema educativo español, en relación con la enseñanza obligatoria, se detectan como problemas más acuciantes las altas tasas de abandono escolar prematuro. De acuerdo con Faci (2011), éste deber ser definido como las personas de 18 a 24 años que han cursado como máximo el primer ciclo de educación secundaria y no siguen ninguna educación o formación complementarias. Esta situación genera una elevada proporción de trabajadores poco cualificados entre la población adulta (RIQUELME, 2006; MELENDRO, 2011).

Asimismo, los altos índices de repetidores generan desigualdad y aumentan los costes de la educación. El hecho de repetir curso aumenta el riesgo de abandono escolar, reduce la realización de las expectativas (ESPAÑA, 2012) y multiplica las desigualdades educativas, lo que incide en la carrera profesional y en la remuneración salarial.

No obstante, el rendimiento de los alumnos en la escuela es estable y se sitúa en torno a la media de la Unión Europea (UE), constatándose algunas mejoras. Según el Programa para la Evaluación Internacional de Alumnos de la Organización para la Cooperación y el Desarrollo Económicos (OCDE) de 2012 (ESPAÑA, 2012), el rendimiento 
de los alumnos españoles mejoró en comprensión lectora y matemáticas. Ya los resultados del Estudio Internacional de Tendencias en Matemáticas y Ciencias (ESPAÑA, 2015) ponen de manifiesto que los alumnos de $4^{\circ}$ de Educación Primaria en España han mejorado su rendimiento en comprensión lectora y matemáticas en estos últimos cinco años.

Los problemas evidenciados hasta ahora en nuestro sistema educativo y en otros países (CABRERA; CABRERA, 2008) han obligado a los responsables en política educativa a buscar, generar e instrumentar medidas de mayor impacto. Una de estas medidas es la adopción de programas acción tutorial, orientación educativa, atención a la diversidad y autoevaluación en cada centro escolar. Estos son aspectos fundamentales, dentro de la supervisión que realiza la inspección educativa española. Los estudios consultados inciden, por un lado, en la necesidad de realizar una revisión de sus funciones y quehaceres para que ambos estén alineados en un cometido más evaluador, a la vez sugieren una revisión de la visita al aula para que ésta contribuya a la mejora educativa (CAMACHO, 2015) y, por otro se plantea la necesidad de realizar una planificación homologada de una serie de actuaciones que contribuya al éxito educativo de todo el alumnado, generando dinámicas de trabajo colaborativo en el seno de los equipos de inspección (ROMER0, 2017).

A este respecto, Ehren y otros autores (2013) definen una serie de acciones que están contribuyendo a la mejora de la calidad en diferentes sistemas educativos (Países Bajos, Inglaterra, Suecia, Irlanda, Austria y la República Checa). Entre ellas destacan:

- Propiciar una autoevaluación de calidad por parte de los centros educativos para mejorar sus sistemas internos de evaluación y autoevaluación. Para ello se establecen mecanismos internos que garantizan su calidad, lo que genera un ciclo de mejora y rendición de cuentas.

- Difusión de buenas prácticas y estándares de calidad como ejemplos que guien la acción de los centros.

- Realización de propuestas de mejora por parte de la inspección a los centros tras las inspecciones y obligación de las escuelas de su puesta en práctica.

- Comunicación de los resultados de las inspecciones escolares a los miembros de la comunidad educativa (padres, alumnado, responsables políticos locales) como partes implicadas en la mejora de la enseñanza.

- Implicación de los directivos de los centros escolares en el proceso de supervisión escolar.

\section{Los modelos de inspección}

Centrándonos en el entorno de los países comunitarios, por ser los más próximos cultural y políticamente, encontramos que no es posible el establecimiento de un marco común de la Inspección Educativa en la Unión Europea, dado que la supervisión de los centros educativos varía de un Estado a otro (EHREN et al., 2013).

Algunos países contemplan la existencia de un Cuerpo de Inspección que tienen como función la supervisión y evaluación de los centros. En Italia y España, la responsabilidad corresponde exclusivamente a la Inspección, aunque sea una responsabilidad compartida 
entre el Estado Central y las Comunidades Autónomas. En nuestro país, cada Comunidad Autónoma dispone de una Inspección Educativa y existe una Inspección estatal (la Alta Inspección) con unas funciones básicamente de homologación.

En otros países, no se contempla la existencia de un cuerpo específico que realice estas funciones. En países como Dinamarca, son asesores académicos los que atienden y aconsejan al profesorado; en otros casos, las corporaciones locales supervisan la labor del director del centro a la vez que asesoran a los profesores. Son los directores de los centros los que tienen las funciones de la supervisión pedagógica.

Existen sistemas educativos en los que la supervisión de los centros es compartida entre los inspectores y otros órganos vinculados a los departamentos de la administración educativa, o autoridades educativas.

Resulta evidente que todas las administraciones educativas afrontan las tareas de supervisión (realizada sobre la comunidad escolar para orientarla sobre la mejora de la calidad de la enseñanza), control (para exigir el cumplimiento de la normativa legal vigente y así garantizar el derecho a la educación de toda la ciudadanía) y evaluación (sobre la organización y el funcionamiento de los centros y del profesorado) para la mejora del sistema educativo correspondiente. Como hemos señalado, la forma en que estas funciones son organizadas es variable, aunque todos los países mantienen algún tipo de control del funcionamiento del sistema educativo, de los centros y demás servicios que lo integran.

Los diferentes sistemas de inspección en Europa están convergiendo hacia modelos que buscan la mejora de la calidad educativa y proponen un enfoque de supervisión de los centros escolares integrando instrumentos cualitativos y cuantitativos (HALL, 2017a), propiciando el diálogo con los directivos de los centros para definir responsabilidades y estándares básicos que favorezcan el cumplimiento de la normativa e impulsen la autoevaluación de los centros (HALL, 2017b) como agentes responsables y protagonistas de su propia mejora (BAXTER; CLARKE, 2013; BROWN et al., 2018).

En esta línea, Hult y Segerholm (2016) exponen que en el nuevo modelo de supervisión y asesoramiento de la inspección educativa en Suecia está adquiriendo una creciente importancia la evaluación de los centros centrada en áreas estrechamente vinculadas al cumplimiento del marco normativo. Ello se debe, en parte, a que la acción de la inspección escolar se está convirtiendo en muchos países europeos en una respuesta de los responsables políticos ante la necesidad de mejorar los logros escolares (LAWN; GREK, 2012; GREK; LINDGREN, 2015). Costa y Pires (2011) manifiestan que las políticas transnacionales europeas se están influenciando unas a otras al centrar los modelos de supervisión educativa en la mejora de los logros escolares, comparando los resultados obtenidos con los de otros países, así como elaborando estándares e indicadores de calidad. A la vez que se propicia un modelo de inspección menos duro e inquisitivo.

No obstante, aunque el objetivo de todas las inspecciones escolares es la mejora de la calidad de la educación, el propio concepto de lo que es una educación de calidad varía de un país a otro; oscilando desde las perspectivas relacionadas con la equidad para proporcionar igualdad de oportunidades a todos los estudiantes, hasta modelos basados en indicadores sobre la calidad de la enseñanza y el aprendizaje inspirados en la eficacia 
escolar. Así mismo, los indicadores de calidad de la educación pueden variar en función del ámbito sobre el que se ejerce la función inspectora. Por ello, existen planteamientos que se centran en mejorar la calidad de todo el sistema educativo y otros que ponen el foco de atención en los centros escolares individualmente.

\section{El Plan de la Inspección Educativa de Andalucía}

El Plan General de Actuación para el periodo 2016-2019 (ANDALUCÍA, 2016a) ha fijado como finalidad contribuir al éxito educativo del alumnado, favorecer su permanencia en el sistema y garantizar el ejercicio de los derechos y el cumplimiento de los deberes de la comunidad educativa. Para ello es necesario definir claramente las características del modelo de inspección educativa en Andalucía en cuanto a su planteamiento organizativo, definición de actuaciones prioritarias y recursos que pretende integrar. En este sentido, es imprescindible que las actuaciones que se implementen en un modelo de inspección educativa se incardinen dentro de un marco legal, así como en un modelo de intervención y metodologías de trabajo (KATIPOGLU, 2016).

El Plan de Actuación Trienal queda estructurado en las siguientes cuatro actuaciones, referidas al alumnado de las enseñanzas obligatorias, que son desarrolladas a través de acciones concretas e implementadas con recursos para su desarrollo (ANDALUCÍA, 2016a, 2016b).

1. Intervención centrada en la atención personalizada del alumnado en riesgo de no obtener la titulación básica, a través de medidas preventivas y recuperadoras que eviten la ruptura de los procesos de aprendizaje o la reincorporación al sistema, mediante la supervisión de factores clave adecuados a dicha intervención.

Esta actuación tiene entre sus prioridades la supervisión del desarrollo de medidas preventivas y recuperadoras que eviten la ruptura de los procesos de aprendizaje, la valoración de la organización de las medidas de seguimiento y control del absentismo y/o el abandono escolar implantada por los centros.

2. Supervisión y asesoramiento de la organización y funcionamiento de los centros para contribuir al éxito educativo y a la prevención del abandono escolar.

La actuación se planifica y desarrolla en las distintas etapas, enseñanzas y modalidades, atendiendo entre otros aspectos a la supervisión y asesoramiento de inicio de curso, análisis de resultados del alumnado e indicadores de centros, Supervisión y asesoramiento a equipos directivos, supervisión de las memorias de autoevaluación y planes de mejora elaborados por los centros.

3. Intervención en procesos de evaluación y autoevaluación: selección y evaluación de la función directiva; evaluación del funcionariado docente en prácticas; evaluación de servicios y programas; participación en evaluaciones externas del sistema educativo; evaluación del alumnado y garantías procedimentales.

Este conjunto de acciones implica entre otras actividades, como recogida y análisis de información y comunicación entre los implicados en relación con cada una de las tareas de supervisión y evaluación a desarrollar, las cuales abarcan entre otras a la evaluación de la función directiva y los procesos de selección de directivos de los centros, así como 
la evaluación de los funcionarios docentes en periodo de prácticas o el desarrollo de las medidas que propician las garantías del proceso de evaluación del alumnado.

4. Supervisión y asesoramiento de la garantía del ejercicio de los derechos y deberes de la comunidad educativa, de la participación, la igualdad y la convivencia.

Esta última actuación tiene como objeto garantizar el ejercicio de los derechos y deberes de todos los miembros de la comunidad educativa, especialmente, en lo que respecta al seguimiento de la gestión de la convivencia en los centros escolares, la acción tutorial y la participación de los diferentes miembros de la comunidad educativa en la vida escolar de los centros.

El modelo de intervención de la inspección de educación en la Comunidad Autónoma de Andalucía se desarrolla a través de unas características, unos elementos metodológicos y aspectos organizativos contenidos en lo que Luna (2014) define como sus señas de identidad. Este modelo se desarrolla con el objetivo de cohesionar y homologar todas las actuaciones del Plan General de Actuación, generando dinámicas de cambio en sus aspectos organizativos y en las pautas de actuación de la inspección.

Los principios de organización y funcionamiento que sostienen el sistema de trabajo en red son: horizontalidad, accesibilidad, funcionalidad, trabajo en equipo, descentralización, autonomía profesional, participación y multidireccionalidad.

Una de las sinergias que posibilitan estas dinámicas de comunicación, colaboración e innovación o cambio es lo que en la literatura se viene denominando trabajo en red. Según Ball y Junemann (2012) existe una creciente literatura sobre la influencia de las interconexiones y el trabajo de redes de expertos que promueven el consenso cognitivo. Un ejemplo, nos lo ofrecen Grek y Ozga (2010) al considerar que este modelo favorece un sistema de supervisión más interconectado, menos burocrático, más flexible e interregional. En este sentido, Alcalá (2016) afirma que el inspector debe liderar un trabajo colaborativo con otros agentes externos que proporcione a los centros el apoyo necesario para la búsqueda de proyectos educativos innovadores que supongan la transformación curricular y metodológica en las aulas, integrando recursos motivadores y adecuados.

Por ello, los Servicios de Inspección se están convirtiendo, cada vez más, en entornos colaborativos (OZGA, 2009) donde el trabajo en equipo preside buena parte de las actuaciones.

En este entorno, es necesario que la inspección educativa propicie la inclusión de las TIC en su quehacer diario y entre los miembros de la comunidad educativa para establecer una relación más interactiva, continuada y dinámica con los centros de enseñanza, y no sólo con sus directores, sino también con los demás miembros del equipo directivo e incluso con los profesores. Por lo que sin duda la capacitación digital de los inspectores está marcando las relaciones con los centros que inspeccionan (SOLER FIEREZ, 2015). Por ello en el Servicio de inspección de Andalucía se fomenta la integración de diversos recursos tecnológicos (ROMERO; MARTÍNEZ-SERRANO, 2017; ROMERO, 2017, 2018), siendo el más reciente y novedoso la creación de la plataforma Inspectio. 


\section{Objetivos}

El objetivo de esta investigación es conocer la valoración de los inspectores de educación sobre su contribución a la mejora de los logros escolares del alumnado, a través de la puesta en práctica del plan de inspección. De manera más específica, este trabajo pretende averiguar si existen diferencias significativas en la percepción de estos profesionales en función de una serie de variables personales de identificación (género, formación, edad, situación laboral, responsabilidad en coordinación de actuaciones y/o áreas).

\section{Metodología}

El enfoque metodológico seguido ha sido de corte cuantitativo, empleando para ello un diseño descriptivo no experimental y no correlacional, es decir se comienza efectuando una investigación descriptiva del estado del tema, donde la realidad se presenta como algo externo al investigador y la finalidad es conocerla y explicarla para poder realizar conclusiones y aportaciones que permitan mejorarla. Según Hernández, Fernández y Baptista (2009) la investigación descriptiva busca especificar propiedades, características y rasgos importantes de cualquier fenómeno que se analice.

\section{Muestra}

La población de nuestro estudio está formada por los inspectores e inspectoras miembros del Servicio de Inspección educativa en las diferentes Delegaciones Territoriales de Educación que conforman las ocho provincias de la Comunidad Autónoma de Andalucía, compuesta por un total 250 miembros (no obstante estas plantillas se han visto incrementadas por el Acuerdo $^{2}$ de 29 de marzo de 2007 entre la Consejería y las Organizaciones Sindicales, para la mejora del funcionamiento de los centros por la aplicación de la LEA). El tipo de muestreo utilizado es el no probabilístico-incidental, que es aquel en el cual el investigador selecciona directa e intencionadamente la muestra, debido a que tiene fácil acceso a la misma y es representativa de la población (SABARIEG0, 2004). La muestra $(n=98)$ obtenida representa el 35,5 \% del total de la población.

La muestra se caracteriza por una superioridad, en lo que a representación se refiere, del sexo masculino (77,6\%), aunque el sexo femenino también está bien representado $(22,4 \%)$. La distribución por sexos en la muestra es proporcional a la distribución de hombres y mujeres en los servicios de inspección educativa. En relación con la edad, destaca el segmento de inspectores e inspectoras que tienen entre 51 y 60 años, dado que suponen prácticamente la mitad de la muestra (48\%); seguido por el intervalo de más de 60 años con un 27,6\% de representación. El grupo de participantes que cuentan entre 41 y 50 años supone 18,4\% de la muestra. El sector menos representado son los miembros de menos de 41 años con un 6,1\% del total. En lo relacionado con la formación previa

2- En el apartado octavo de dicho acuerdo se recoge: Las plantillas de Inspectores de educación se incrementarán hasta conseguir, en el período 2008/2012, una relación 1/15 entre el número de inspectores e inspectoras y el de centros docentes (ANDALUCÍA, 2007, p. 64-69). 
al ingreso en el cuerpo, predomina la especialidad de Orientación Educativa (34,7\%), seguidos por los licenciados en Humanidades o de las Ciencias Sociales y Jurídicas (31\%), Ciencias de la actividad física y del deporte (8,2\%), Música y danza (6,10\%), Lenguas extranjeras $(3,1 \%)$ y por último un $1 \%$ a otros.

En cuanto a la experiencia, los participantes mayoritarios son los que tienen uno de entre 3 a 6 cursos (35,7\%), seguidos de los que tienen más de 11 cursos (31,6\%), los que han permanecido entre 7 y 11 años (25,5\%), y por último los que tienen menos de 2 cursos de permanencia en el cuerpo $(7,1 \%)$. Por último, en relación con la responsabilidad de coordinación en áreas o actuaciones del plan de inspección, predominan los que no tienen ninguna responsabilidad (57,1\%), así como los que no pertenecen al equipo de coordinación provincial $(79,6)$. No se consideró adecuado establecer de forma precisa la muestra por provincias para asegurar el anonimato o evitar la generación de suspicacias en cuanto a diferencias de aplicación del Plan de Inspección en los diferentes Servicios Provinciales de Inspección.

\section{Procedimiento}

La investigación se encaminó, inicialmente, a la construcción de un instrumento que permitiera recoger la opinión de los inspectores de educación sobre su contribución a la mejora de los logros escolares del alumnado de las enseñanzas básicas, a través de la puesta en práctica del plan de inspección.

La primera fase de construcción del cuestionario tipo escala Likert se inició con un proceso de revisión bibliográfica para extraer un conjunto de indicadores que sirvieran de referencia a la elaboración de los ítems que integraron el cuestionario. A continuación, se realizó la validación del primer borrador del instrumento mediante un sistema de jueces (cuatro expertos). El juicio de expertos se define como una opinión informada de personas con trayectoria en el tema, que son reconocidas por otros como expertos cualificados en éste, y que pueden dar información, evidencia, juicios y valoraciones (ESCOBAR; CUERVO, 2008). Los jueces llevaron a cabo una valoración ítem a ítem siguiendo cuatro criterios (DORANTES; HERNÁNDEZ; TOBÓN, 2016): suficiencia, claridad, coherencia y relevancia, valorando de 0 a 10 su adecuación. Una vez confeccionado el cuestionario, tras la primera validación, procedimos a enviarlo por correo o entregar en mano, según la ubicación de los Servicios Provinciales de Inspección. Esta fase se desarrolló entre los meses de marzo y junio de 2018.

\section{Análisis de datos}

Los datos fueron analizados con el paquete informático SPSS para Windows, versión 15.0. Para conocer los objetivos de nuestra investigación, se realizó un análisis de los ítems a través de frecuencias y porcentajes de cada uno de los ítems del cuestionario, así como un análisis descriptivo (medias y desviaciones típicas). Para estudiar los objetivos específicos, en función de las variables sociodemográficas, se empleó un análisis de varianza (ANOVA), acompañado de comparaciones múltiples a posteriori (prueba Scheffé) 
y de la información sobre el tamaño del efecto (siguiendo los criterios de Jacob Cohen, 1988). En todos los análisis se ha empleado un nivel de confianza del 95\% (significación $\mathrm{p}<0,05)$.

\section{Resultados}

La escala se sometió al análisis de fiabilidad global, el nivel de consistencia interna, optando por el alfa de Cronbach (.886) que se considera alto. Así mismo, el análisis de Spearman-Brown también corrobora dicha consistencia, arrojando valores $(0,862$ y 0,805) que nos indican una alta correlación. Por último, se calculan los estadísticos de viabilidad para los elementos que la componen, con la finalidad de analizar el poder de discriminación de cada uno de ellos. Los valores obtenidos, nos indican valores iguales o superiores a 0,881 , por lo que el cuestionario presenta un elevado grado de consistencia interna. Para conocer la correlación de variables se utilizó la prueba de esfericidad de Barlett y la medida de adecuación muestral KMO (Kaiser-Meyer-Olkin). Los valores nos indicaron la pertinencia de realizar el análisis factorial $(\mathrm{KM}=, 764$; KMO: Chi-cuadrado=1210,564; gl=351; Sig.=,000). Posteriormente se realizó la determinación de las comunalidades, esto es, la proporción de varianza explicada por los factores comunes. Las comunalidades iniciales son siempre igual a uno. Los valores oscilaron entre 0,515 (item 5) y 0,810 (ítem 22). Para realizar el análisis factorial se utilizó el método de extracción de análisis de componentes principales y rotación Varimax. Dicho análisis revela la convergencia de cuatro factores que explican el 51,954 de la varianza.

La escala quedó estructurada en los ítems de identificación expuestos (sexo, edad, formación, coordinación de áreas y/o actuaciones, situación administrativa y pertenencia al equipo de coordinación provincial) y 27 preguntas tipo Likert con cuatro opciones de respuesta en un contínuum ( 1 =nada; $2=$ poco; 3 =bastante; y $4=$ mucho). Quedando estructurado en cuatro dimensiones: atención a los miembros de la comunidad educativa (9 ítems), supervisión de la orientación y acción tutorial (7 items), atención e inclusión a la diversidad (6 ítems) y recursos tecnológicos ( 5 ítems). En este artículo nos centraremos en la primera dimensión: atención a los miembros de la comunidad educativa $(=0,855)$.

El análisis de los datos nos revela que la actuación más valorada en la relativa a las entrevistas y reuniones con equipos directivos y responsables de órganos de coordinación didáctica $(3,14)$, seguida por las entrevistas con los responsables de la orientación educativa en los centros $(3,04)$. El resto de las acciones reciben puntuaciones por debajo de tres: las visitas a las aulas y entrevistas con el profesorado $(2,98)$, el seguimiento de la incidentalidad $(2,90)$, la supervisión del Programa de Tránsito entre etapas educativas $(2,88)$, el asesoramiento al profesorado para la atención a la diversidad y a necesidades educativas especiales $(2,73)$, la supervisión del programa de orientación y acción tutorial (POAT) (2,67), la elaboración, presentación y remisión a los centros de los informes homologados de las diferentes actuaciones $(2,64)$ y las entrevistas con padres y madres o sus representantes $(2,55)$. La desviación típica de estos ítems se sitúa entre 0,60 y 0,69 (Tabla 1). 
Tabla 1- Medias, desviación típica y carga factorial. Atención miembros comunidad

\begin{tabular}{|c|c|c|c|}
\hline \multicolumn{4}{|l|}{ Atención a los miembros de la comunidad educativa } \\
\hline Ítems ( $\mathrm{M}=2,83 ; \mathrm{DT}=0,64)$ & M & DT & Carga Factorial \\
\hline Visitas a las aulas y entrevistas con el profesorado. & 2,98 & 0,60 & 0,712 \\
\hline Supervisión del POAT. & 2,67 & 0,63 & 0,634 \\
\hline Supervisión del Programa de Tránsito entre etapas educativas. & 2,88 & 0,67 & 0,557 \\
\hline Entrevistas y reuniones con equipos directivos y responsables de órganos de coordinación didáctica. & 3,14 & 0,60 & 0,717 \\
\hline Entrevistas con los responsables de la orientación educativa en los centros. & 3,04 & 0,65 & 0,737 \\
\hline $\begin{array}{l}\text { Entrevistas con padres y madres o sus representantes (delegados, miembros de C.E. y } \\
\text { directivos de AMPAS). }\end{array}$ & 2,55 & 0,62 & 0,671 \\
\hline $\begin{array}{l}\text { Elaboración, presentación y remisión a los centros de los informes homologados de las } \\
\text { diferentes actuaciones. }\end{array}$ & 2,64 & 0,69 & 0,603 \\
\hline Seguimiento de la incidentalidad. & 2,90 & 0,69 & 0,602 \\
\hline $\begin{array}{c}\text { Asesoramiento al profesorado para la atención a la diversidad y a necesidades educativas } \\
\text { especiales. }\end{array}$ & 2,73 & 0,63 & 0,439 \\
\hline
\end{tabular}

Fuente: Elaboración propia.

La opinión de la inspección educativa sobre su contribución a la mejora de los logros escolares del alumnado (a través de la puesta en práctica del plan de inspección relacionada con la atención a los miembros de la comunidad educativa) son: la atención directa basada en entrevistas, tanto con los equipos directivos $(87,7 \%)$ como con los responsables de la orientación educativa (82,6\%), así como las visitas a las aulas y las entrevistas con el profesorado (80,6\%). También se valora positivamente la supervisión al programa de tránsito entre las etapas educativas (74,5\%) y el asesoramiento al profesorado para la mejora de la atención a la diversidad (68,20\%). Por último, las actuaciones menos valoradas son: el seguimiento de la incidentalidad (67,4\%), la presentación de informes homologados a los centros $(66,2 \%)$, la supervisión del programa de orientación y acción tutorial (POAT) (62,3\%), y las entrevistas con las familias (52\%). 
Tabla 2- Porcentajes. Atención miembros comunidad educativa

\begin{tabular}{|c|c|c|c|c|}
\hline \multicolumn{5}{|l|}{ Atención a los miembros de la comunidad educativa } \\
\hline Ítems & Nada & Poco & Bastante & Mucho \\
\hline Visitas a las aulas y entrevistas con el profesorado. & $0 \%$ & $19,4 \%$ & $63,3 \%$ & $17,3 \%$ \\
\hline Supervisión del POAT. & $2 \%$ & $35,7 \%$ & $55,1 \%$ & $7,2 \%$ \\
\hline Supervisión del Programa de Tránsito entre etapas educativas. & $2 \%$ & $23,5 \%$ & $51,2 \%$ & $23,3 \%$ \\
\hline $\begin{array}{l}\text { Entrevistas y reuniones con equipos directivos y responsables de órganos de } \\
\text { coordinación didáctica. }\end{array}$ & $0 \%$ & $12.2 \%$ & $61,2 \%$ & $26,5 \%$ \\
\hline Entrevistas con los responsables de la orientación educativa en los centros. & $1,1 \%$ & $16,3 \%$ & $60,2 \%$ & $22,4 \%$ \\
\hline $\begin{array}{l}\text { Entrevistas con padres y madres o sus representantes (delegados, miembros de } \\
\text { C.E. y directivos de AMPAS). }\end{array}$ & $2,1 \%$ & $45,9 \%$ & $46,9 \%$ & $5,1 \%$ \\
\hline $\begin{array}{l}\text { Elaboración, presentación y remisión a los centros de los informes homologados } \\
\text { de las diferentes actuaciones. }\end{array}$ & $3,1 \%$ & $30,6 \%$ & $55,1 \%$ & $11,2 \%$ \\
\hline Seguimiento de la incidentalidad. & $2 \%$ & $30,6 \%$ & $42,9 \%$ & $24,5 \%$ \\
\hline $\begin{array}{c}\text { Asesoramiento al profesorado para la atención a la diversidad y a necesidades } \\
\text { educativas especiales. }\end{array}$ & $1,2 \%$ & $30,6 \%$ & $62,1 \%$ & $6,1 \%$ \\
\hline
\end{tabular}

Fuente: Elaboración propia.

Para medir las diferencias en la percepción de los miembros del servicio de inspección educativa sobre los efectos de su labor en la mejora de los logros escolares (a través de la atención a los miembros de la comunidad educativa) en las variables personales de identificación consideradas, se examinan las medias, desviaciones típicas y significación (tabla 1). El análisis de varianza no ha aportado diferencias significativas en las variables: género $\left(\mathrm{F}_{1,22}=0,12 ; \mathrm{p}=0,728\right)$, edad $\left(\mathrm{F}_{2,97}=2,36 ; \mathrm{p}=0,07\right)$, formación $\left(\mathrm{F}_{5,04}=1,01 ; \mathrm{p}\right.$ $=0,42)$, ser miembro del equipo de Coordinación Provincial $\left(\mathrm{F}_{1,80}=0,561 ; \mathrm{p}=0,45\right) \mathrm{y}$ situación administrativa $\left(\mathrm{F}_{1,80}=0,29 ; \mathrm{p}=0,58\right)$. Sin embargo, ser responsable de alguna de las actuaciones del Plan Anual o de Áreas Estructurales $\left(\mathrm{F}_{1,57}=7,91 ; \mathrm{p}=0,006\right)$ es fuente significativa de variación entre los que son coordinadores y los que no (tamaño del efecto mediano $d=0,79$ ). Las diferencias encontradas en relación con la realización de tareas de coordinación de áreas estructurales o actuaciones del plan puede deberse a que ello requiere el desarrollo de funciones diferenciadas con respecto a los miembros del servicio, así como la participación en determinadas actividades de formación. 
María del Carmen Martínez SERRANO

Tabla 3- Diferencias significativas

\begin{tabular}{|c|c|c|c|c|c|}
\hline \multicolumn{6}{|c|}{ Atención a los miembros de la comunidad educativa } \\
\hline Variables & Media & $\mathrm{DE}$ & $\mathrm{F}$ & $\mathrm{p}$ & $d$ \\
\hline $\begin{array}{c}\text { Hombre } \\
\text { Mujer }\end{array}$ & $\begin{array}{l}25,56 \\
25,90\end{array}$ & $\begin{array}{l}4,20 \\
3,57\end{array}$ & 0,121 & 0,728 & 0,08 \\
\hline $\begin{array}{l}\text { Menos de } 41 \text { años } \\
\text { De } 41 \text { a } 50 \text { años } \\
\text { De } 51 \text { a } 60 \text { años } \\
\text { Más de } 60 \text { años }\end{array}$ & $\begin{array}{l}24,33 \\
23,66 \\
26,02 \\
26,59\end{array}$ & $\begin{array}{l}3,01 \\
5,08 \\
3,95 \\
3,26\end{array}$ & 2,36 & 0,07 & $\begin{array}{l}0,16 \\
0,48 \\
0,72 \\
0,51 \\
0,68 \\
0,15\end{array}$ \\
\hline Área de Humanidades o de las CCSS y Jurídicas & 25,48 & 3,38 & 1,01 & 0,42 & 0,52 \\
\hline Área de Ciencias Experimentales y de la Salud & 27,60 & 4,55 & & & 0,27 \\
\hline Ingeniero o Arquitecto & 27,20 & 3,49 & & & $\begin{array}{l}0,24 \\
0,06\end{array}$ \\
\hline Lenguas Extranjeras & 24,66 & 2,51 & & & 0,09 \\
\hline Educación Musical, Danza o Arte Dramático & 24,50 & 6,56 & & & 0,59 \\
\hline Ciencias de la Actividad Física y del Deporte & 24,50 & 4,40 & & & $\begin{array}{l}0,43 \\
1,99\end{array}$ \\
\hline Orientación Educativa & 25,73 & 4,02 & & & \\
\hline Otros & 19,00 & 4,05 & & & \\
\hline Coordinación Sí & 26,92 & 3,44 & 7,91 & 0,006 & 0,79 \\
\hline Coordinación No & 24,67 & 4,23 & & & \\
\hline Miembro Sí & 26,25 & 3,98 & 0,561 & 0,45 & 0,19 \\
\hline Miembro No & 25,48 & 4,08 & & & \\
\hline Provisional & 25,2 & 3,79 & 0,29 & 0,58 & 0,13 \\
\hline Funcionario & 25,75 & 4,13 & & & \\
\hline
\end{tabular}

Fuente: Elaboración propia. 


\section{Discusión y conclusiones}

La mejora de los logros escolares es una labor conjunta y organizada de todos los sectores que integran un sistema educativo. El enfoque de la medición utilizada se ha centrado prioritariamente en el conjunto de la comunidad educativa (padres, profesores y alumnado), excluyendo el trabajo efectuado por los miembros del servicio de inspección educativa. De ahí que consideremos necesario realizar un análisis sobre el trabajo realizado por la inspección educativa para fomentar la mejora de los resultados escolares, ya que tiene entre sus funciones garantizar el cumplimiento en la práctica de los principios recogidos en la ley de educación. La supervisión del sistema educativo debe realizarse de forma que permita atender las necesidades educativas de todo el alumnado de educación obligatoria, garantizando la calidad, equidad, flexibilidad e igualdad, independientemente de sus características o circunstancias (DOMÍNGUEZ; LÓPEZ; VÁZQUEZ, 2016; MARSHALL, 2008).

La inspección escolar es utilizada en la mayoría de los sistemas educativos europeos como un importante instrumento para el control y promoción de la calidad de las escuelas (DEDERING; SOWADA, 2016; MONARCA; FERNÁNDEZ, 2016; RÖNNBERG, 2012). En esta línea, Ehren (2010) considera que la intervención de la inspección escolar consigue mejoras significativas en la calidad de la educación cuando su acción ayuda a definir las debilidades, genera retroalimentación con los centros supervisados y consensua acuerdos con los equipos directivos sobre los aspectos a mejorar.

Los inspectores valoran que las entrevistas, tanto con los responsables de la puesta en práctica de la acción tutorial y la orientación educativa y profesional (profesorado, orientadores y miembros de los equipos de orientación educativa), como con los miembros de los equipos directivos y jefaturas de departamento y otros responsables de los órganos de coordinación didáctica (HAEP; BEHNKE; STEINS, 2016), junto con las visitas a las aulas son las acciones habituales de supervisión con más capacidad de ejercer efectos positivos en la mejora de los logros escolares. Lo que sugiere que se percibe como un factor relevante, en términos generales, es la presencia del inspector o inspectora de referencia en el centro, ya sea visitando aulas o manteniendo reuniones con el profesorado, en general, o con el equipo directivo (CAMACHO, 2015).

Asimismo, los datos aportados por este estudio reflejan la utilidad que tienen las reuniones y contactos que mantienen los inspectores de educación con los responsables de los centros y las familias, en relación con la mejora la acción tutorial y la orientación educativa y vocacional, ya sea en el desarrollo de actuaciones homologadas o en el seguimiento de la incidentalidad. En este sentido, son numerosos los estudios realizados que avalan la importancia de profundizar en este aspecto para mejorar el clima de la comunidad educativa (ETXEBERRIA; INTXAUSTI; AZPILLAGA, 2017), la importancia de la acción tutorial y la interacción escuela- familia en el logro académico (LUZARRAGA; NÚÑEZ; ETXEBERRIA, 2018).

Es muy sensato reflexionar que, dado el tamaño de la muestra, los resultados hallados no son completamente extrapolables al resto de la población, por tanto, considerando esta limitación, sería recomendable realizar estudios más amplios que abarquen otras comunidades autónomas, así como a otros países para recoger la valoración que los 
inspectores realizan sobre su contribución a la mejora de los logros escolares y concretar aportaciones que contribuyan a la mejora de los planes de inspección.

\section{Referencias}

ALCALÁ, María Lourdes. ¿Debe ser la inspección impulsora de la Innovación en los centros educativos? Revista Avances en Supervisión Educativa, Valladolid, n. 26, p. 1-29, 2016. Disponible en: <https://avances. adide.org/index.php/ase/article/view/578>. Acceso en: 24 feb. 2017.

ANDALUCÍA. Acuerdo de 29 de marzo de 2007, entre la Consejería y las Organizaciones Sindicales, para la mejora del funcionamiento de los centros por la aplicación de la Ley de Educación de Andalucía. Sevilla: [s. n.], 2007. Disponible en: <https://www.feandalucia.cco0.es/plantillai.aspx?p=29\&d=681. Acceso en: 23 febr. 2020.

ANDALUCÍA. Orden de 26 de julio de 2016, por la que se establece el Plan General de actuación de la Inspección Educativa de Andalucía para el período 2016-2019. Boletín Oficial de la Junta de Andalucía, Sevilla, n. 154, p. 11-52, 2016a. Disponible en: <https://www.juntadeandalucia.es/boja/2016/154/1>. Acceso en: 26 jun. 2017.

ANDALUCÍA. Resolución de 29 de agosto de 2016, de la Viceconsejería, por la que se aprueban las instrucciones para el desarrollo, la dirección y la coordinación del Plan General de Actuación de la Inspección Educativa de Andalucía para el curso escolar 2016-2017. Boletín Oficial de la Junta de Andalucía, Sevilla, n. 173, p. 7-22, 2016b. Disponible en: <https://www.juntadeandalucia.es/boja/2016/173/1>. Acceso en: 26 jun. 2017.

BALL, Stephen J.; JUNEMANN, Carolina. Networks, new governance and education. Bristol: The Policy Press, 2012.

BAXTER, Jacqueline; CLARKE, John. Farewell to the tick box inspector? Ofsted and the changing regime of school inspection in England. Oxford Review of Education, Oxford, v. 39, n. 5, p. 702-718, 2013.

BROWN, Martin et al. Integrated co-professional evaluation? Converging approaches to school evaluation across frontiers. Australian Journal of Teacher Education, Joondalup, v. 43, n. 12, p. 76-90, 2018.

CABRERA, Leopoldo José; CABRERA, Blas. Heterogeneidad de centros y desigualdades de rendimiento educativo. Tempora, La Laguna, n. 11, p. 61-99, 2008.

CAMACHO, Alexandre. La visita de inspección, su función malherida. Reice, Madrid, v. 13, n. 4, p.79-91, 2015.

COHEN, Jacob. Statistical power analysis for the behavioral science. 2. ed. New York: Lawrence Erlsbaun, 1988. Disponible en: <http://www.utstat.toronto.edu/ brunner/oldclass/378f16/readings/CohenPower. pdf>. Acceso en: 28 abr. 2016.

COSTA, Estela; PIRES, Ana Marcia. The role of the European inspections in the European educational space-echoes from Portugal regarding the assessment of schools. Research in Higher Education Journal, Georgia, n. 13, p.1-10, 2011. 
DEDERING, Kathrin; SOWADA, Moritz G. Reaching a conclusion_-procedures and processes of judgement formation in school inspection teams. Educational Assessment, Evaluation and Accountability, Cham, v. 29, n. 1, p.5-22, 2016.

DOMÍNGUEZ, José; LÓPEZ, Antonio; VÁZQUEZ, Elia. Atención a la diversidad en la educación secundaria obligatoria: Análisis desde la inspección educativa. Aula Abierta, Oviedo, n. 44, p.70-76, 2016.

DORANTES, Judith Araceli; HERNÁNDEZ, José Silvano; TOBÓN, Sergio. Juicio de expertos para la validación de un instrumento de medición del síndrome de Burnout en la docencia. Ra Ximhai, México, DC, v. 12, n. 6, p. 327-346, 2016.

EHREN, Melanie Catharina Margaretha. Effecten van toezicht op het basisonderwijs. Pedagogische Studiën, Amsterdã, n. 87, p. 165-182, 2010.

EHREN, Melanie Catharina Margaretha et al. Impact of school inspections on improvement of schoolsdescribing assumptions on causal mechanisms in six European countries. Educational Assessment, Evaluation and Accountability, Cham, v. 25, n. 1, p. 3-43, 2013.

ESCOBAR, Jazmine; CUERVO Angela. Validez de contenido y juicio de expertos: una aproximación a su utilización. Avances de Medición, Bogotá, n. 6, p. 27-36, 2008.

ESPAÑA. Ministerio de Educación, Cultura y Deporte. Estudio Internacional de Tendencias en Matemáticas y Ciencias (TIMSS). Madrid: Instituto Nacional de Evaluación Educativa, 2015. Disponible en: <http://blog.intef. es/inee/2016/11/23/estudio-internacional-de-tendencias-en-matematicas-y-ciencias-timss-2015/>. Acceso en: 9 nov. 2019.

ESPAÑA. Ministerio de Educación, Cultura y Deporte. Panorama de la educación: indicadores de la OCDE 2012. Informe español. Madrid: Ministerio de Educación, Cultura y Deporte, 2012.

ETXEBERRIA, Feli; INTXAUSTI, Nahia; AZPILLAGA, Veronica. School climate in highly effective schools in the autonomous region of the Basque Country (Spain). Reice, Madrid, v. 15, n. 4, p. 5-26, 2017.

EUROSTAT. Informe Eurostat. Informe sobre España. Bruselas: Eurostat-Comisión Europea, 2017. Disponible en: $\quad<$ https://ec.europa.eu/info/sites/info/files/2017-european-semester-country-report-spain-es.pdf>. Acceso en: 20 oct. 2018.

$\mathrm{FACl}$, Fernando. El abandono escolar prematuro en España. Avances en Supervisión Educativa, Valladolid, $\mathrm{n}$. 14, p. 1-26, 2011. Disponible en: <http://www.adide.org/revista/images/stories/pdf_14/ase14_monog03. pdf>. Acceso en: 16 jun. 2017.

GREK, Sotiria; LINDGREN, Joakim (Ed.). Governing by inspection. London: Routledge, 2015.

GREK, Sotiria; OZGA, Jenny. Re-inventing public education: the new role of knowledge in education policymaking. Public Policy and Administration, New York, v. 25, n. 3, p. 271-288, 2010. 
HAEP, Anna; BEHNKE, Kristin; STEIN, Gisela. Classroom observation as an instrument for school development: school principal perspectives on its relevance and problems. Studies in Educational Evaluation, Amberes, n. 49, p. 1-6, 2016.

HALL, Jeffrey Brooks. Examining school inspectors and education directors within the organisation of school inspection policy: perceptions and views. Scandinavian Journal of Educational Research, 0slo, v. 61, n. 1, p. 112-126, 2017b.

HALL, Jeffry Brooks. "Governing by templates" through new modes of school inspection in Norway. Journal Education Change, Boston, v. 18, n. 3, p. 161-182, 2017a.

HERNÁNDEZ, Roberto; FERNÁNDEZ, Carlos; BAPTISTA, María del Pilar. Metodología de la investigación. México, DC: Mc Graw-Hill, 2009. Disponible en: <https://www.esup.edu.pe/descargas/dep_investigacion/ Metodologia\%20de\%20la\%20investigaci\%C3\%B3n\%205ta\%20Edici\%C3\%B3n.pdf>. Acceso en: 22 de dic. 2017.

HULT, Agneta; SEGERHOLM, Christina. The process of juridification of school inspection in Sweden. Utbildning \& Demokrati, Örebro, v. 25, n. 2, p. 95-118, 2016.

KATIPOGLU, Ilke. Ministry of National Education Inspectors' adoption level of risk-based inspection model and the consideration of its applicability at schools. Eurasian Journal of Educational Research, Ankara, n. 66, p. 407-428, 2016.

LAWN, Martin; GREK, Sotiria. Europeanizing education: governing a new policy space. Oxford: Symposion Books, 2012.

LUNA, Pedro Ángel. Las señas de identidad del modelo de intervención de la inspección de educación de Andalucía. Avances en Supervisión Educativa, Valladolid, n. 21, p. 1-9, 2014. Disponible en: <https:// avances.adide.org/index.php/ase/article/view/82>. Acceso en: 18 agto. 2014.

LUZARRAGA, Jon Mikel; NúÑEZ, Juan Manuel; ETXEBERRIA, Juan. Análisis de las expectativas de los centros de Bachillerato de alta y baja eficacia escolar: percepción de la inspección educativa. Revista Complutense de Educación, Madrid, v. 29, n. 4, p. 1075-1090, 2018.

MARSHALL, Chris. Thirty-five years of school inspection: raising educational standards for children with additional needs? British Journal of Special Education, Reino Unido, v. 35, n. 2, p. 69-77, 2008.

MELENDR0, Miguel. El tránsito a la vida adulta de los jóvenes en dificultad social: la incidencia de la intervención socioeducativa y la perspectiva de profesionales y empresarios. Revista de Educación, Madrid, n. 356, p. 327-352, 2011.

MONARCA, Héctor; FERNÁNDEZ-GONZÁLEZ, Noelia. El papel de la inspección educativa en los procesos de cambio. Cadernos de Pesquisa, São Paulo, v. 46, n. 159, p. 212-233, 2016.

OZGA, Jenny. Governing education through data in England: from regulation to self-evaluation. Journal of Education Policy, Brighton, v. 24, n. 2, p. 149-163, 2009. 
RIQUELME, Graciela Clotilde. La relación entre educación y trabajo: continuidad, rupturas y desafíos. Anales de la Educación Común, Buenos Aires, v. 2, n. 5, p. 68-75, 2006. Disponible en: <http://servicios.abc.gov. ar/lainstitucion/revistacomponents/revista /archivos/anales/numero05/archivosparaimprimir/8.riquelme. pdf>. Acceso en: 15 abr. 2007.

ROMERO, Manuel Ángel. La comunicación mediada por tecnología como base de la mejora de la actuación de la inspección educativa: el Plan de Inspección 2016-17 en Andalucía. Revista Avances en Supervisión Educativa, Valladolid, n. 27, p. 1-25, 2017. Disponible en: <https://avances.adide.org/index.php/ase/ article/view/587>. Acceso en: 21 jul. 2017.

ROMERO, Manuel Ángel. Uso de las TIC por la inspección educativa en Andalucía. Revista de Educación Mediática y TIC, Córdoba, v. 7, n. 1, p. 275-295, 2018. Disponible en: <http://www.uco.es/servicios/ ucopress/ojs/index.php/edmetic/article/view/9969>. Acceso en: 14 sept. 2018.

ROMERO, Manuel Ángel; MARTíNEZ-SERRANO, María del Carmen. Inclusión de los medios tecnológicos en el Plan de Inspección de Andalucía. Riite, Murcia, n. 2, p. 41-53, 2017. Disponible en: <http://revistas. um.es/riite/article/view/295121>. Acceso en: 31 jul. 2017.

RÖNNBERG, Linda. Reinstating national school inspections in Sweden: the return of the state. Nordic Studies in Education, Noruega, v. 32, n. 2, p. 69-83, 2012.

SABARIEGO, Marta. El proceso de investigación. In: BISQUERRA, Rafael (Coord.). Metodología de la investigación educativa. Madrid: La Muralla, 2004. p. 127-163.

SOLER FIERREZ, Eduardo. Decálogo de las competencias profesionales del inspector de educación. Reice, Madrid, v. 13, n. 4, p. 149-160, 2015.

Recibido en: 11.05.2019 Revisado en: 22.10.2019 Aprobado en: 02.12.2019

María del Carmen Martínez Serrano es doctora en Pedagogía por la Universidad de Granada. Profesora titular del área de Didáctica y Organización Escolar, Departamento de Pedagogía, de la Universidad de Jaén, Facultad de Humanidades y Ciencias de la Educación. 